\title{
Controle de temperatura de um forno mufla
}

\author{
Guilherme P. C. de Miranda* Daniel de A. Fernandes ** \\ * Departamento de Engenharia de Produção e Mecânica, \\ Universidade Federal de Juiz de Fora (UFJF), Juiz de Fora, MG, \\ (e-mail: guilherme.pires@engenharia.ufjf.br) \\ ** Departamento de Energia Elétrica, \\ Universidade Federal de Juiz de Fora (UFJF), Juiz de Fora, MG, \\ (e-mail:daniel.fernandes@ufjf.edu.br)
}

\begin{abstract}
This paper presents a nonlinear temperature controller developed for a muffle furnace available in the Manufacturing Process Laboratory of the Faculty of Engineering of the Federal University of Juiz de Fora (UFJF) in Brazil. The furnace is used in the heat treatment of ferrous and non-ferrous materials. The core of the proposed model-based, digital controller is a PI controller. Other control techniques are also employed to complete it. A simplified model of the plant was constructed and validated based on real data obtained in full-scale trials. Many full-scale trials were also conducted in order to validate the performance of the closed-loop system. Experimental results are presented and discussed herein. A stability proof is given to attest to the robustness of the closed-loop system. The temperature controller was implemented through the free hardware electronic prototyping platform called Arduino BT. It was tuned offline through computer simulations based on the Twiddle algorithm.

Resumo: Este artigo apresenta um controlador não linear de temperatura desenvolvido para um forno mufla disponível no Laboratório de Processos de Fabricação da Faculdade de Engenharia da UFJF. O forno é empregado no tratamento térmico de materiais ferrosos e não ferrosos. O controlador digital proposto tem como núcleo um controlador do tipo PI, acrescido de outras técnicas de controle, e foi desenvolvido com base no modelo da planta controlada. Um modelo simplificado da planta foi construído e validado através de dados reais obtidos em ensaios práticos. O sistema de controle em malha fechada também foi bastante testado e, assim, validado; resultados experimentais são mostrados e discutidos. Uma prova de estabilidade é dada para atestar a robustez da estabilidade do sistema em malha fechada. A implementação foi realizada através da plataforma de prototipagem eletrônica de hardware livre Arduino BT. A sintonia foi feita offline, através de simulação computacional utilizando o algoritmo Twiddle.
\end{abstract}

Keywords: Muffle furnace; Nonlinear control; PID control; Process control; Temperature control.

Palavras-chave: Controle de processo; Controle de temperatura; Controle não linear; Controle PID; Forno mufla.

\section{INTRODUÇÃO}

\subsection{Introdução}

Este artigo apresenta um controlador de temperatura proposto para um forno mufla. Tais fornos são equipamentos de médio porte, um tipo de estufa para altas temperaturas $\left(\leqslant 1300^{\circ} \mathrm{C}\right)$. São utilizados em diversos tipos de aplicações, normalmente de laboratório. A maior parte dos modelos comerciais utiliza resistências elétricas para elevar a temperatura no interior de suas câmaras de aquecimento e materiais refratários na isolação térmica dessas câmaras.

Este trabalho dedica-se especialmente ao forno mufla modelo $2000-1-220 \mathrm{~V}_{\mathrm{CA}}$ e $3,96 \mathrm{~kW}$ nominais - , fabricante nacional Zezimaq, empregado no tratamento térmico de diversos materiais ferrosos e não ferrosos no Laboratório de Processos de Fabricação da Faculdade de Engenharia da UFJF. A Figura 1 mostra uma imagem do forno mufla.
Deseja-se que os materiais sob tratamento térmico sejam aquecidos a taxas controladas, além de serem mantidos sob temperaturas elevadas durante intervalos de tempo precisos, para terem suas microestruturas modificadas conforme descrevem as teorias respectivas e, dessa forma, terem modificadas suas propriedades físico-químicas e mecânicas (Krauss, 2015; Brooks, 1982). Um bom e breve resumo da importância do tratamento térmico, bem como de sua automatização, pode ser encontrado na introdução do trabalho de Zambaldi et al. (2015), por exemplo.

O primeiro autor é Técnico Administrativo em Educação (TAE) no referido laboratório. Ele identificou a necessidade dos usuários de controlar a temperatura do forno em rampas e patamares, além de assegurar menores erros de temperatura durante os tratamentos térmicos realizados.

O controlador proposto deve substituir o controlador originalmente fornecido com o forno mufla, também mostrado 


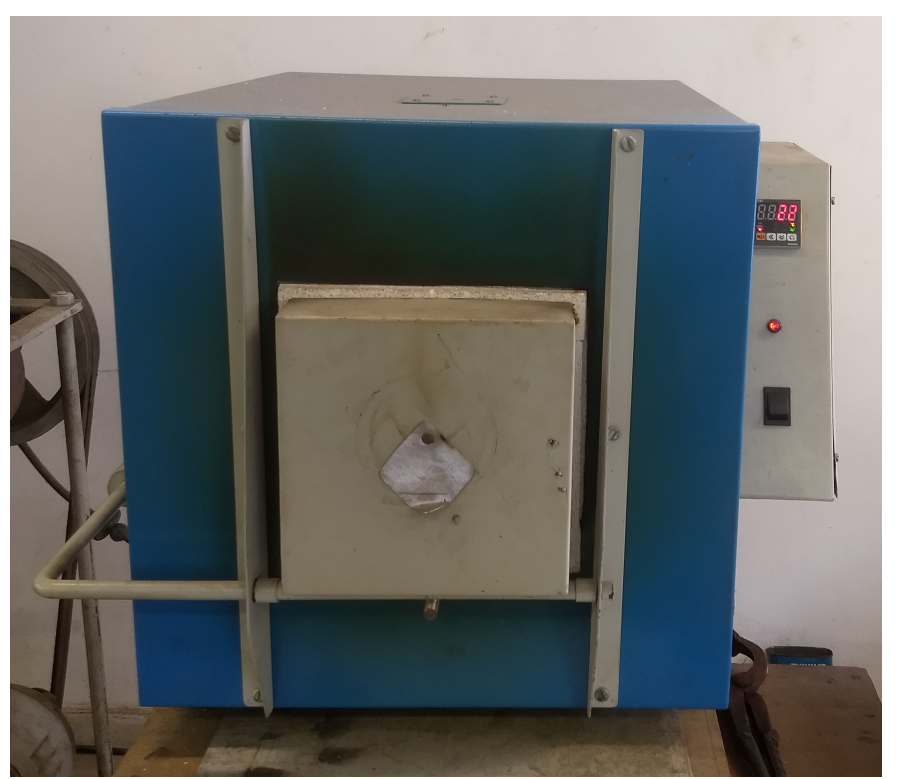

Figura 1. Forno mufla, modelo 2000-1, fabricado pela empresa nacional Zezimaq.

na Figura 1, que implementa apenas um controle básico do tipo $O N-O F F$. Ele é baseado em um controlador do tipo Proporcional-Integral (PI) (Åström \& Hägglund, 2011; Franklin et al., 2009; Ogata, 2009). Outras técnicas de controle são utilizadas conjuntamente para assegurar um desempenho satisfatório em Malha Fechada (MF). O controlador é de tempo discreto, robusto, de baixo custo e de fácil implementação. A topologia do controlador foi definida com base na literatura e na experiência profissional dos autores. Uma prova de estabilidade é dada para atestar a robustez da estabilidade do sistema em MF. Muitos ensaios foram realizados para verifiar a robustez do desempenho do sistema em MF.

O restante do artigo é organizado da seguinte forma: a Seção 2 apresenta o modelo da planta e o controlador proposto; a Seção 3 apresenta alguns dos resultados experimentais obtidos; a Seção 4 discute os resultados experimentais apresentados; por fim, na Seção 5, são apresentadas as conclusões e são dadas algumas sugestões de trabalhos futuros.

\subsection{Objetivo geral}

O objetivo deste trabalho é apresentar um controlador de temperatura para o referido forno que seja robusto em termos de estabilidade e desempenho, de baixo custo e fácil de implementar.

\subsection{Revisão bibliográfica}

Muitas são as aplicações de controle automático voltadas para equipamentos de laboratório e industriais em geral. Elas se prestam a propósitos nobres como reduzir o consumo de energia, economizar tempo de processamento, aumentar a segurança e a qualidade dos produtos manufaturados, assegurar a repetibilidade dos processos de manufatura, entre outros. Alguns exemplos de trabalhos dedicados aos fornos elétricos são Ramirez-Laboreo et al. (2016), Hambali et al. (2014) e Neacă (2012). Outros exemplos que merecem destaque são comentados abaixo.
Zambaldi (2016) desenvolveu controladores do tipo Proporcional-Integral-Derivativo (PID) para controlar fornos utilizados no tratamento térmico de aços. Seu objetivo foi diminuir subjetivamente a interferência humana no processo de tratamento térmico. Para modelar a planta foi ajustada uma Função de Transferência (FT) de primeira ordem com atraso de transporte, prática comum na área de controle de sistemas térmicos. De posse da FT, diferentes métodos de sintonia foram utilizados nos controladores PID até que se obteve um desempenho satisfatório.

No trabalho de Guerra (2006) diversas FTs foram ajustadas e validadas a partir das curvas de resposta em Malha Aberta (MA) levantadas para uma série de 11 valores da tensão elétrica $\left(20 \mathrm{~V}_{\mathrm{CA}}\right.$ a $\left.220 \mathrm{~V}_{\mathrm{CA}}\right)$ aplicada às resistências de aquecimento de um forno. Foi então definida como modelo, por comparação dos diagramas dos lugares das raízes gerados em cada caso, a FT referente a $20 \mathrm{~V}_{\mathrm{CA}}$. O atraso de transporte foi substituído por um aproximante (racional) de Padé de ordem [1/1]. Três controladores da família PID foram então sintetizados, também com base no método do lugar das raízes, e posteriormente implementados e testados na prática. Os controladores PI e PID garantiram desempenhos satisfatórios e semelhantes entre si.

Em Serapião (2016) foram validados experimentalmente, e também comparados entre si em termos de desempenho e capacidade de lidar com as incertezas do sistema, três técnicas avançadas de controle para um forno elétrico: i) "controle baseado em regras", ii) lógica nebulosa - fuzzy logic, em inglês - do tipo 1 e iii) lógica nebulosa do tipo 2. A terceira delas mostrou-se um pouco superior às demais. Vale destacar que, ainda outra vez, a planta foi modelada à maneira tradicional na área, ou seja, através de uma FT de primeira ordem com atraso de transporte, porém com parâmetros variáveis descritos por funções polinomiais em função da tensão elétrica de alimentação das resistências de aquecimento.

\section{CONTROLADOR}

\subsection{Modelagem e validação do modelo da planta}

O modelo da planta é indispensável no processo de desenvolvimento do controlador pretendido. E um bom modelo neste caso é aquele que apresenta um compromisso satisfatório entre a complexidade e a capacidade de descrever matematicamente as principais características da dinâmica da planta (Franklin et al., 2009; Ogata, 2009; Garcia, 2005; Egeland \& Gravdahl, 2002).

Plantas como o forno mufla mostrado na Figura 1 possuem um único atuador - conjunto de resistências de aquecimento - , incapaz de arrefecê-lo. O arrefecimento ocorre passivamente pela inevitável perda de calor para o ambiente, neste caso benéfica e útil, através da imperfeita isolação térmica das paredes do forno. Isto implica, na prática, que somente pequenos sobressinais $(\leqslant 5 \%)$ podem ser tolerados na operação em MF. Também ocorre um atraso de transporte, porque o calor radiante não provoca imediata elevação da temperatura dos materiais no interior do forno, nem do termopar instalado no poço termométrico - mais detalhes são fornecidos na Subseção 3.1. Entretanto, não ocorre atraso de transporte significativo no arrefecimento causado pela perda de calor para o ambiente. Informações 
detalhadas sobre o assunto podem ser encontradas, por exemplo, em Garcia (2005) e Egeland \& Gravdahl (2002).

A partir da análise de muitas curvas de resposta ao degrau em MA, obteve-se o seguinte modelo no espaço de estados:

$$
\left\{\begin{array}{l}
\frac{d}{d t}[\theta(t)]=-\tau^{-1} \theta(t)+\alpha\left(1-\beta \exp \left(-\gamma \frac{u(t)}{100}\right)\right), \\
T_{\mathrm{cam}}(t)=T_{\mathrm{amb}}+\max \left(0 ; \tau^{-1} \theta(t)\right)
\end{array}\right.
$$

onde $\alpha \approx 1074,21669, \beta \approx 1,14600154$ e $\gamma \approx 5,76890357$ são constantes, $\tau \approx 11030$ s é uma constante de tempo, $u(t)$ [\%] corresponde à razão cíclica - Duty Cycle (DC), em inglês - da modulação por largura de pulso - Pulse-Width Modulation (PWM), em inglês —, técnica utilizada para controlar a potência média fornecida ao conjunto de resistências de aquecimento do forno - mais detalhes são fornecidos na Subseção $3.1-, T_{\text {cam }}(t)\left[{ }^{\circ} \mathrm{C}\right]$ é a temperatura na câmara de aquecimento e $T_{\mathrm{amb}}\left[{ }^{\circ} \mathrm{C}\right]$ é a temperatura ambiente externa ao forno. Note-se em (1) a dinâmica linear de primeira ordem tipicamente adotada na modelagem de sistemas térmicos (Franklin et al., 2009; Ogata, 2009; Garcia, 2005; Egeland \& Gravdahl, 2002).

Importa saber que o modelo matemático em (1)

I. descreve de forma simplificada apenas as principais características da dinâmica da planta; ele é válido exclusivamente nos intervalos $0 \% \leqslant u(t) \leqslant 100 \%$ e $T_{\text {amb }} \leqslant T_{\text {cam }}(t)$, considerado o regime de operação normal do forno;

II. ignora propositalmente o atraso de transporte $(\approx 100 \mathrm{~s})$ e as imperfeições do sensor de temperatura utilizado $\left(\right.$ erro $\left.<1^{\circ} \mathrm{C}\right)$, pois a influência de ambos na operação automática do forno torna-se irrelevante na prática;

III. leva em consideração, através dos valores ajustados das constantes $\alpha, \beta$ e $\gamma$, a perda de calor para o ambiente: a temperatura diminui quando $u(t)<2,3623 \%$.

Os valores de $\alpha, \beta, \gamma$ e $\tau$ foram ajustados através de métodos numéricos convenientes para isto. $\mathrm{O}$ modelo em (1) pode ser considerado satisfatório e validado a partir da apreciação das curvas mostradas na Figura 2, curvas essas obtidas para operação da planta em MA. Os erros máximos são todos inferiores a $4 \%$ dos valores finais desejados.

\subsection{Controlador}

Um controlador digital — mais detalhes são fornecidos na Subseção 3.1 - é preferido nesta aplicação devido à simplicidade de implementação e de se fazer futuras atualizações. Como ele apresenta uma taxa de amostragem elevada $(1 \mathrm{~Hz})$ em relação à bem mais lenta dinâmica da planta, a influência da operação do controlador em tempo discreto tanto na estabilidade - atraso de fase - quanto no desempenho do sistema em MF pode ser desprezada. Logo, a fim de simplificar a notação, o controlador é aqui descrito em tempo contínuo, embora ele opere realmente em tempo discreto.

O controlador PI

$$
u_{\mathrm{pi}}(t)=k_{p} e(t)+k_{i} \int_{0}^{t} e(\lambda) d \lambda
$$

constitui o núcleo do controlador todo, onde $k_{p}, k_{i} \in \mathbb{R}_{>0}$ são respectivamente os ganhos proporcional e integral, e

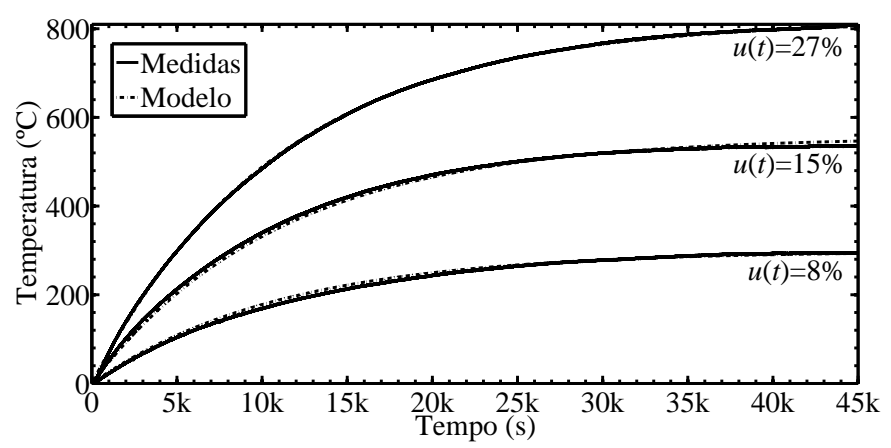

Figura 2. Validação do modelo matemático em (1) através do uso de três razões cíclicas diferentes.

$e(t)$ é o erro atuante dado por

$$
e(t)=T_{\text {des }}(t)-T_{\text {cam }}(t)
$$

onde $T_{\text {des }}(t)$ [ ${ }^{\circ} \mathrm{C}$ ] é o sinal de referência de temperatura. Ele pode tanto ser constante - patamar de temperatura - quanto variar linearmente - rampa de aquecimento. O algoritmo trapezoidal, ou de Tustin, (Franklin et al., 2009; Ogata, 2009; Garcia, 2005; Egeland \& Gravdahl, 2002) foi escolhido para implementar a integral presente em (2) devido à sua estabilidade e ao bom compromisso entre exatidão e simplicidade que apresenta.

$\mathrm{O}$ sinal de controle aplicado à planta é

$$
u(t)=-\left(\frac{100}{\gamma}\right) \ln \left(\frac{\alpha-\operatorname{sat}\left(u_{\mathrm{pi}}(t)\right)}{\alpha \beta}\right),
$$

onde a função saturação é definida como

$$
\operatorname{sat}\left(u_{\mathrm{pi}}(t)\right):=\left\{\begin{array}{lll}
0 & \Longleftrightarrow u_{\mathrm{pi}}(t) \leqslant 0 \\
u_{\mathrm{pi}}(t) & \Longleftrightarrow 0<u_{\mathrm{pi}}(t)<\eta \alpha, \\
\eta \alpha & \Longleftrightarrow u_{\mathrm{pi}}(t) \geqslant \eta \alpha
\end{array}\right.
$$

onde $\eta=0,996420830925$.

Para completar o controlador proposto - esquematizado na Figura 3 -, uma estratégia também simples é implementada para prevenir o carregamento excessivo do integrador - integrator wind-up, em inglês — do controlador PI quando ocorre a saturação do sinal de controle. Isso é essencial para assegurar um bom desempenho do sistema em MF (Åström \& Hägglund, 2011; Franklin et al., 2009; Ogata, 2009). Enquanto $u_{\mathrm{pi}}(t) \geqslant \eta \alpha$, o valor de $\int_{0}^{t} e(\lambda) d \lambda$ é mantido inalterado. A utilização do controlador digital possibilita fazer tal implementação de forma muito simples e confiável.

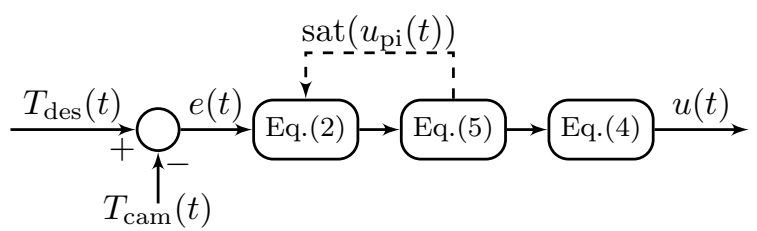

Figura 3. Diagrama de blocos completo do controlador proposto. O forno mufla recebe $u(t)$ e gera $T_{\text {cam }}(t)$. A geração do sinal de referência $T_{\text {des }}(t)$ é aqui omitida apenas para simplificar o diagrama.

\subsection{Prova de estabilidade}

Uma prova da estabilidade, no sentido de Lyapunov (Franklin et al., 2009; Slotine \& Li, 2005; Khalil, 2002), do sistema em MF é oferecida nesta subseção. 
Teorema 1. (Estabilidade da MF). Seja (1) a planta controlada. Seja (4) o sinal de controle para a planta, produzido pela combinação de (2)-(3) e (5). Sejam os ganhos $k_{p}, k_{i} \in \mathbb{R}_{>0}$. Então, o único ponto de equilíbrio $e^{*}=0-$ origem do espaço de erros $\mathcal{E} \subseteq \mathbb{R}$ - é Globalmente Exponencialmente Estável (GEE) e a saída $T_{\text {cam }}(t)$ apresenta erro nulo de regime permanente para sinais de referência em degrau.

Prova. Para $T_{\text {des }}(t)>T_{\mathrm{amb}}$, a substituição em (3) da equação de saída do modelo matemático em (1) resulta em $e(t)=\left(T_{\mathrm{des}}(t)-T_{\mathrm{amb}}\right)-\tau^{-1} \theta(t)$, cuja derivada no tempo é $\frac{d}{d t}[e(t)]=\frac{d}{d t}\left[T_{\text {des }}(t)\right]-\tau^{-1} \frac{d}{d t}[\theta(t)]$. Com a substituição da equação da dinâmica do modelo matemático em (1) nesta derivada, realizada somente após a substituição de (4) na equação da dinâmica do modelo matemático em (1), a equação diferencial não linear e homogênea $\frac{d}{d t}[e(t)]=$ $-\tau^{-1}\left(e(t)+\operatorname{sat}\left(k_{p} e(t)+k_{i} \int_{0}^{t} e(\lambda) d \lambda\right)\right)$ é obtida no espaço de erros $\mathcal{E}$. Seja a função de Lyapunov candidata $V(e(t)):=$ $0,5 e^{2}(t)$, positiva definida e radialmente ilimitada. Sua derivada ao longo da trajetória do erro $\left\langle\nabla V(e(t)), \frac{d}{d t}[e(t)]\right\rangle=$ $-\tau^{-1} e^{2}(t)-e(t) f(e(t)) \leqslant-\tau^{-1}|e(t)|^{2}<0, \forall e(t) \neq 0$, onde $f(e(t)):=\tau^{-1} \operatorname{sat}\left(k_{p} e(t)+k_{i} \int_{0}^{t} e(\lambda) d \lambda\right)$, tal que $e(t) \leqslant 0$ $\Longrightarrow f(e(t))=0$ e $e(t)>0 \Longrightarrow f(e(t))>0$, conforme $(5)$.

\subsection{Sintonia do controlador}

O algoritmo Twiddle (Thrun et al., 2006) mostra-se uma solução simples para o desafio de sintonizar o controlador PI satisfatoriamente sem poder lançar mão das técnicas usualmente empregadas no tratamento de sistemas Lineares e Invariantes no Tempo (LITs) - alocação dos polos dominantes de MF. Há, entretanto, várias outras soluções disponíveis além desse algoritmo que foi escolhido.

O Twiddle busca encontrar valores (sub)ótimos para os ganhos $k_{p}, k_{i}>0$ através de sucessivas simulações computacionais sem ter conhecimento do sistema em MF nem a necessidade de calcular gradientes de quaisquer funções. A parada do algoritmo ocorre tão logo o primeiro par de ganhos (sub)ótimos é encontrado. Não há, contudo, a garantia de convergência do algoritmo.

Neste caso, a parada do algoritmo ocorreu na primeira vez em que foi produzida uma resposta cujo sobressinal pertencia ao intervalo 1-5\%, com uma taxa de elevação da temperatura que pertencia ao intervalo $0,1-0,2^{\circ} \mathrm{C} / \mathrm{s}$, simultaneamente.

\section{RESULTADOS EXPERIMENTAIS}

\subsection{Materiais e métodos}

Para modelar a planta, e posteriormente implementar e testar o controlador de temperatura proposto, foram utilizados os seguintes componentes, mostrados na Figura 4:

1 - (Forno mufla) Termopar tipo K. Junção bimetálica Cromel-Alumel de baixo custo e uso geral para medição de temperaturas no intervalo $-200-1200^{\circ} \mathrm{C}$. Fica alojado no poço termométrico;

2 - Circuito Integrado (CI) dedicado MAX6675. Converte o sinal analógico gerado pelo termopar tipo

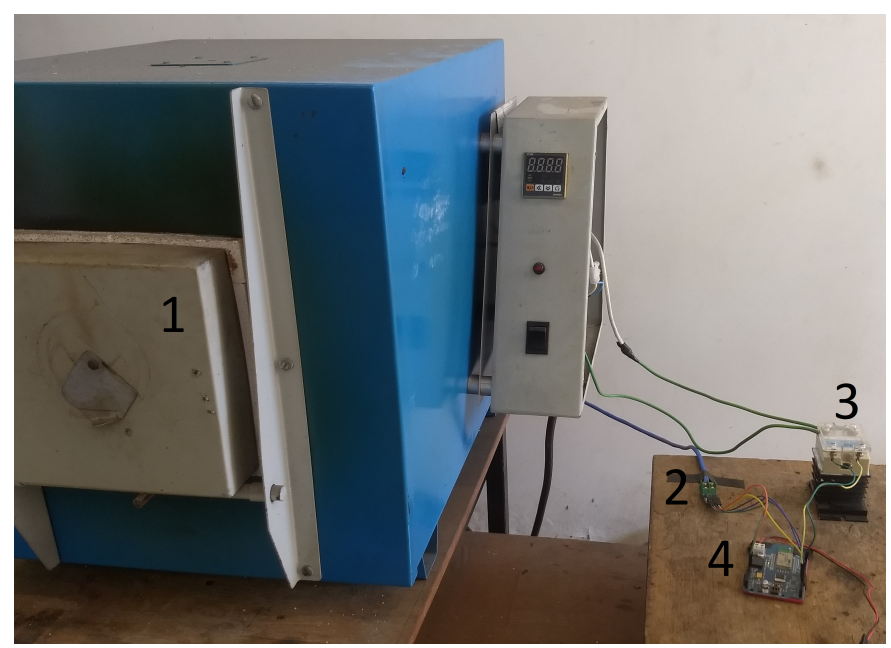

Figura 4. Arranjo utilizado para modelar a planta e testar o controlador proposto.

$\mathrm{K}$ em um sinal digital de resolução de 12 bits $\left(0,25^{\circ} \mathrm{C}\right)$ e o envia ao Arduino BT via comunicação serial com fio;

3 - Relé de Estado Sólido (RES) modelo TZC-40A. Possui um único contato do tipo Normalmente Aberto (NA) para até $40 \mathrm{~A}$, tensão de trabalho até $380 \mathrm{~V}_{\mathrm{CA}}$ e tensão de acionamento na faixa $4-32 \mathrm{~V}_{\mathrm{CC}}$. Alimenta o conjunto de resistências de aquecimento com uma potência média proporcional à razão cíclica do sinal PWM gerado pelo Arduino BT;

4 - Plataforma Arduino BT. Baseia-se no microcontrolador ATMEGA328P e no módulo de comunicação serial Bluetooth ${ }^{\circledR}$ WT11. Recebe um sinal digital correspondente à temperatura da câmara de aquecimento, medida à taxa de $1 \mathrm{~Hz}$, e comanda a alimentação do conjunto de resistências de aquecimento. Comunicase via Bluetooth ${ }^{\circledR}$ com um laptop onde o aplicativo MATLAB ${ }^{\circledR}$ armazena os valores medidos. A operação em MA permite aquisitar dados para posterior análise e obtenção de um modelo conveniente da planta. A operação em MF, sem auxílio do MATLAB ${ }^{\circledR}$, realiza o controle de temperatura almejado.

A Figura 5 mostra esquematicamente como os componentes descritos acima são interconectados.

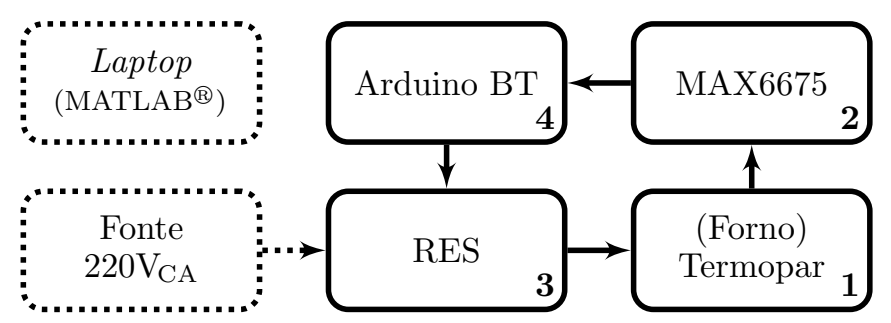

Figura 5. Esquema de interconexão dos componentes descritos nesta subseção e mostrados na Figura 4.

Devido à alta potência nominal do conjunto de resistências de aquecimento do forno $(3,96 \mathrm{~kW})$, não é possível alimentá-lo plenamente, ou seja, utilizar $u(t)=100 \% \mathrm{du}-$ rante o processo de modelagem da planta em MA, e aguardar até que a temperatura na câmara de aquecimento atinja a condição de regime permanente, a fim de proteger a integridade do equipamento. 


\subsection{Resultados experimentais}

Foram realizados numerosos ensaios com o referido forno mufla controlado pelo controlador de temperatura proposto. Resultados experimentais considerados satisfatórios foram alcançados após a conclusão do processo (offline) de sintonia do controlador. Esta subseção apresenta então um dos resultados obtidos em um dos ensaios realizados.

As Figuras 6-9 apresentam os dados coletados no ensaio. A temperatura ambiente registrada no início do ensaio foi $T_{\mathrm{amb}} \approx 24,7^{\circ} \mathrm{C}$. Duas rampas com inclinações distintas $\left(0,1^{\circ} \mathrm{C} / \mathrm{s}\right.$ e $0,17^{\circ} \mathrm{C} / \mathrm{s}$, respectivamente $)$ e dois patamares $\left(450^{\circ} \mathrm{C}\right.$ e $750^{\circ} \mathrm{C}$, respectivamente) foram utilizados como referência $T_{\mathrm{des}}(t)$, exatamente como ocorre tipicamente nos processos de tratamento térmico de interesse.

O erro absoluto máximo de acompanhamento do sinal de referência ocorreu aos $92 \mathrm{~s}\left(|e(t)| \approx 8,2^{\circ} \mathrm{C}\right)$, revela a Figura 7. Fica evidente ali a influência do atraso de transporte da planta. Tal atraso, contudo, foi deliberadamente desprezado neste trabalho, conforme a justificativa dada na Subseção 2.1, uma vez que ele não causa degradação significativa do desempenho do sistema em MF.

O máximo sobressinal ocorrido no caso do primeiro patamar foi de aproximadamente $0,89 \%\left(\approx 4^{\circ} \mathrm{C}\right)$, conforme mostra a Figura 8. O máximo sobressinal ocorrido no caso do segundo patamar foi de aproximadamente $0,6 \%$ $\left(\approx 4,5^{\circ} \mathrm{C}\right)$, conforme mostra a Figura 9. Pode-se inferir facilmente que os erros de regime permanente anulariamse se o sinal de referência permanecesse por mais longo tempo em cada um dos patamares.

\section{DISCUSSÃO}

A análise da resposta do forno mufla apresentada na Subseção 3.2 permite concluir que o controlador proposto satisfaz as especificações de desempenho e estabilidade, ou seja, controla a temperatura da câmara de aquecimento dentro de rígidas tolerâncias, sem grandes sobressinais, porém não nulos propositalmente, de modo a possibilitar altas velocidades de resposta relativamente às variações do sinal de referência $T_{\text {des }}(t)$. Entretanto, os sobressinais observados são inferiores àqueles especificados na Subseção 2.4. Em particular, infere-se que são nulos os erros de regime permanente para entradas de referência em degrau.

A sintonia subótima pode ser melhorada através de mais ou menos numerosa quantidade de tentativas e erros, procedimento que pode ser bastante demorado uma vez que, na prática, somente um ensaio pode ser realizado a cada dia, porque o forno leva muito tempo para arrefecer quando aquecido. A boa notícia é que se pode experimentar valores com base nos ganhos utilizados atualmente $\left(k_{p}=95,95\right.$ e $\left.k_{i}=0,475\right)$ sem instabilizar o sistema, conforme indica a Subseção 2.3.

Pode-se ainda observar que o modelo matemático em (1) está adequado à finalidade à qual se destina. Ele mostrou representar um compromisso satisfatório entre complexidade e síntese da dinâmica da planta modelada. Ele captura somente as principais características da planta constante de tempo $\tau$ e amplitude da resposta à entrada $u(t)$ - e despreza o atraso de transporte inerente a ela.

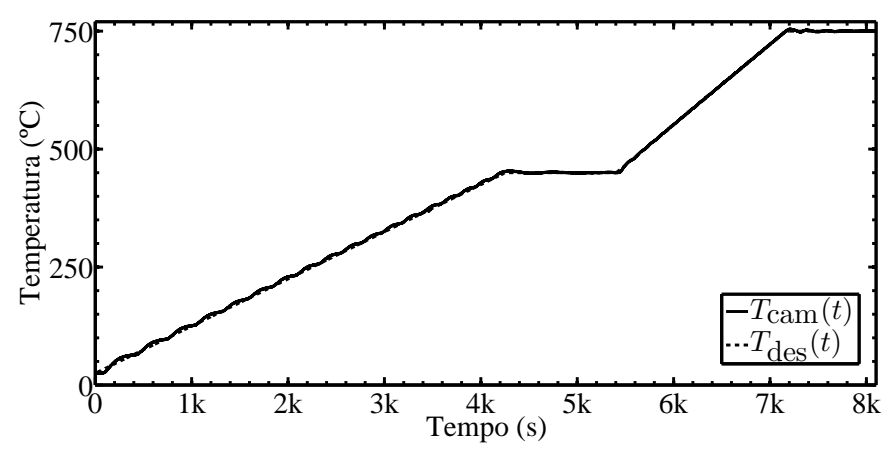

Figura 6. Curva de resposta do forno mufla, típica de um processo de tratamento térmico de interesse.

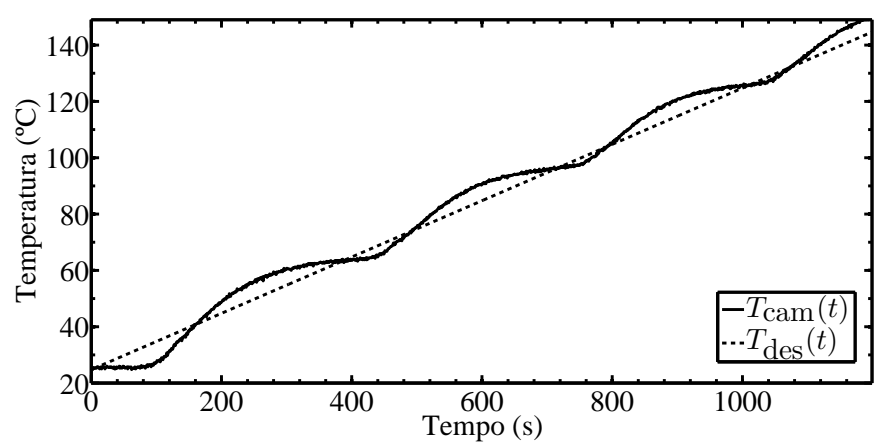

Figura 7. Detalhe da parte inicial da curva de resposta mostrada na Figura 6.

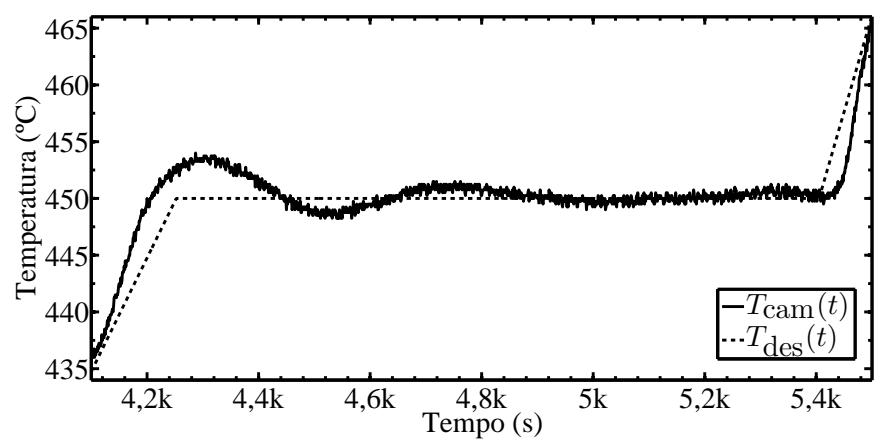

Figura 8. Detalhe do primeiro patamar da curva de resposta mostrada na Figura 6.

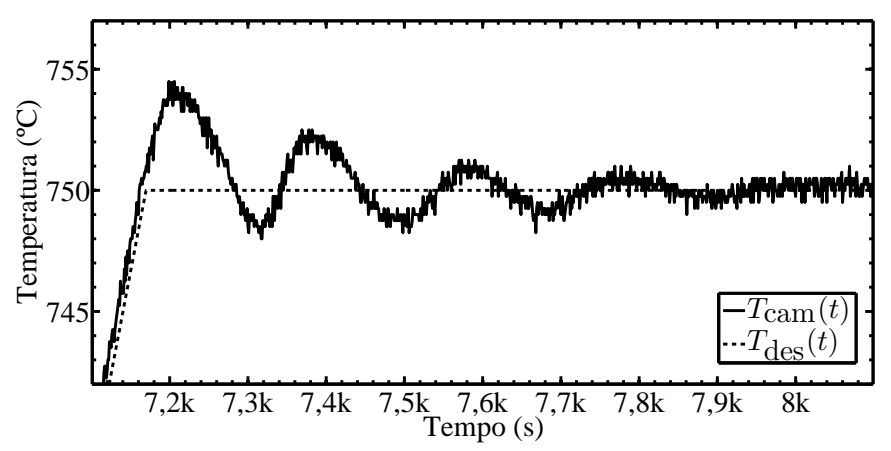

Figura 9. Detalhe do segundo patamar da curva de resposta mostrada na Figura 6 .

\section{CONCLUSÃO}

Este artigo apresentou um controlador digital de temperatura - robusto em termos de estabilidade e desempenho, de baixo custo e de fácil implementação - para um forno mufla disponível no Laboratório de Processos de Fabricação da Faculdade de Engenharia da UFJF. Foram apresen- 
tados os resultados experimentalmente obtidos em um dos muitos ensaios realizados com sucesso, além de uma prova de estabilidade, segundo Lyapunov, para o sistema em MF. As respostas observadas indicam que o forno mufla atende as necessidades dos usuários da universidade de controlar a temperatura em rampas e patamares, além de assegurar menores erros de temperatura durante os tratamentos térmicos realizados.

Trabalhos futuros devem priorizar a melhoria do desempenho do forno através, ou de uma melhor sintonia do controlador ora proposto, ou do desenvolvimento de outro(s) controlador(es), com o cuidado de sintonizá-lo(s) adequadamente. É essencial que algum trabalho futuro tenha o objetivo de desenvolver uma interface homem-máquina baseada em dispositivos móveis — handheld, em inglês como celulares e tablets.

\section{AGRADECIMENTOS}

Os autores agradecem profundamente a colaboração fundamental dos colegas da UFJF, Profs. Exuperry Barros Costa e Leonardo Rocha Olivi.

\section{REFERÊNCIAS}

Åström, K.J. \& Hägglund, T. (2011). PID controllers: theory, design, and tuning. Instrument Society of America, Carolina do Norte, $2^{a}$ edição.

Brooks, C.R. (1982). Heat treatment, structure, and properties of nonferrous alloys. American Society for Metals (ASM International), Novelty.

Egeland, O. \& Gravdahl, J.T. (2002). Modeling and simulation for automatic control. Marine Cybernetics, Trondheim, Noruega.

Franklin, G.F., Powell, J.D., \& Emami-Naeini, A. (2009). Feedback control of dynamic systems. Prentice Hall, Inc., Upper Saddle River, 6 $\underline{a}$ edição.

Garcia, C. (2005). Modelagem e simulação. Editora da Universidade of São Paulo (EDUSP), São Paulo, Brasil, $2^{\underline{a}}$ edição.

Guerra, L.N.A. (2006). Uso de compensador PID no controle da taxa de variação de temperatura em um forno elétrico a resistência. Trabalho de conclusão de curso, Universidade Federal do Rio de Janeiro (UFRJ), Departamento de Engenharia Elétrica.

Hambali, N., Ang, A.A.R., Ishak, A.A., \& Janin, Z. (2014). Various PID controller tuning for air temperature oven system. Em IEEE International Conference on Smart Instrumentation, Measurement and Applications (ICSIMA) 2014 — Malásia. doi:10.1109/ICSIMA.2014. 7047441.

Khalil, H.K. (2002). Nonlinear systems. Prentice Hall, Inc., Upper Saddle River, $3^{\underline{a}}$ edição.

Krauss, G. (2015). Steels: processing, structure, and performance. American Society for Metals (ASM International), Novelty, $2 \stackrel{a}{a}$ edição.

Neacă, A.M. (2012). Comparison between different techniques of controlling the temperature inside a resistive oven. Em IEEE 16th International Conference on System Theory, Control and Computing (ICSTCC) $2012-$ Romênia, 1-6.

Ogata, K. (2009). Modern control engineering. Prentice Hall, Inc., Upper Saddle River, $5 \underline{a}$ edição.
Ramirez-Laboreo, E., Sagues, C., \& Llorente, S. (2016). Dynamic heat and mass transfer model of an electric oven for energy analysis. Applied Thermal Engineering, 93(25), 683-691. doi:10.1016/j.applthermaleng.2015.10. 040.

Serapião, L.A. (2016). Type-2 fuzzy logic system applied to a temperature control of an electric oven. Trabalho de conclusão de curso, Universidade Federal de Juiz de Fora (UFJF), Departamento de Engenharia Mecânica.

Slotine, J.J.E. \& Li, W. (2005). Applied nonlinear control. Pearson Education Taiwan Ltd., Taipei, Taiwan.

Thrun, S., Burgard, W., \& Fox, D. (2006). Probabilistic robotics. MIT Press, Cambridge.

Zambaldi, E.S. (2016). Controle automatizado de fornos para tratamento térmico em aços. Dissertação de mestrado, Universidade Federal de Lavras (UFLA), Departamento de Engenharia de Sistemas e Automação.

Zambaldi, E.S., Magalhães, R.R., Barbosa, B.H.G., Trugilho, P.F., \& Martins, M.B. (2015). Controle automatizado de baixo custo em fornos para tratamentos térmicos em aços. Em I Congresso Mineiro de Engenharia e Tecnologia - Brasil, 562-568. 